\title{
Generalized Orthogonality Relation for Spherical Harmonics
}

\author{
A. Draux ${ }^{1}$ and G. Gouesbet ${ }^{2}$ \\ ${ }^{1}$ Institut National des Sciences Appliquées (INSA) de Rouen, Département de Génie \\ Mathématique, Place Emile Blondel, BP08, 76131, Mont-Saint-Aignan Cédex \\ 2Laboratoire d'Electromagnétisme des Systèmes Particulaires (LESP) Unité Mixte de \\ Recherche (UMR) 6614 du Centre National de la Recherche Scientifique (CNRS) \\ COmplexe de Recherche Interprofessionnel en Aérothermochimie (CORIA) Université de \\ Rouen et Institut National des Sciences Appliquées (INSA) de Rouen BP12, \\ Avenue de l'Université, Technopôle du Madrillet, 76801, Saint-Etienne-du Rouvray
}

France

\section{Introduction}

One of us (G.G), with collaborators, has been involved in the study of generalized Lorenz-Mie theories (GLMTs) describing the interaction between electromagnetic arbitrary shaped beams (typically laser beams) and a class of regular scatterers for which solutions to Maxwell's equations can be found by using the method of separation of variables, e.g. Gouesbet \& Gréhan (2000a), J.A.Lock \& Gouesbet (2009), Gouesbet (2009a), and references therein. It has, at a certain time, been found interesting to examine whether the knowledge gained in the effort of developing electromagnetic GLMTs could be, at least partially, adapted to quantum mechanical problems. The examination of the issue of quantum scattering of quantum arbitrary shaped beams produced several papers devoted to (i) the description of quantum arbitrary shaped beams Gouesbet (2005), Gouesbet \& J.A.Lock (2007) (ii) the evaluation of cross-sections in the case of quantum arbitrary shaped beams interacting with radial quantum potentials Gouesbet (2006a), Gouesbet (2007a) and (iii) the exhibition of formal cross-sectional analogies between electromagnetic and quantum scatterings Gouesbet (2004), Gouesbet (2006b), Gouesbet (2007b). During the development of this work, the evaluation of two integrals, based on spherical harmonics, has been required. These integrals may be obtained from a generalized orthogonality relation for spherical harmonics which is established in this paper. Another recent application concerns the optical theorem and non plane wave scattering in quantum mechanics Gouesbet (2009b).

Section II is devoted to the demonstrations used to reach the generalized orthogonality relation mentioned above. Section III is a conclusion.

\section{Demonstrations}

\subsection{Lemma 1}

Let $X(b)$ denote the following expression :

$$
X(b)=\sin a \sin \theta \cos (\varphi-b)+\cos a \cos \theta
$$


Then :

$$
\int_{0}^{2 \pi} P_{n}(X(b)) e^{-i m(\varphi-b)} d \varphi=\int_{0}^{2 \pi} P_{n}(X(0)) \cos (|m| \varphi) d \varphi
$$

where $P_{n}$ is the Legendre polynomial of degree $n, i=\sqrt{-1}$ and $m \in \mathbf{Z}$.

As a remark for further use, let us insist on the fact that the r.h.s. of Eq.2 does not depend on $b$ nor on the sign of $m$.

\subsection{Proof of Lemma 1}

The integrand $P_{n}(X(b)) e^{-i m(\varphi-b)}$ in the 1.h.s. of Eq.2 is a linear combination of terms reading as :

$$
[\cos (\varphi-b)]^{j} e^{-i m(\varphi-b)}, j=0 \ldots n
$$

Therefore, to demonstrate Eq.2, it is sufficient to prove that :

$$
\int_{0}^{2 \pi}[\cos (\varphi-b)]^{j} e^{-i m(\varphi-b)} d \varphi=\int_{0}^{2 \pi}(\cos \varphi)^{j} \cos (|m| \varphi) d \varphi, \forall j \in \mathbf{N}
$$

Let us introduce a symbol to denote the 1.h.s. of Eq.4, setting :

$$
K_{j m}=\int_{0}^{2 \pi}[\cos (\varphi-b)]^{j} e^{-i m(\varphi-b)} d \varphi
$$

We then make a change of variables from $(\varphi-b)$ to $\Psi$, use the Leibniz formula, and establish:

$$
\begin{aligned}
K_{j m} & =\int_{-b}^{-b+2 \pi}(\cos \Psi)^{j} e^{-i m \Psi} d \Psi \\
& =\frac{1}{2^{j}} \sum_{k=0}^{j}\left(\begin{array}{l}
j \\
k
\end{array}\right) \int_{-b}^{-b+2 \pi} e^{i(2 k-j-m) \Psi} d \Psi \\
& =\frac{2 \pi}{2^{j}}\left(\begin{array}{l}
j \\
k
\end{array}\right) \delta_{0,2 k-j-m}, k=0 \ldots j
\end{aligned}
$$

where $\delta$ denotes the Kronecker symbol.

Let us introduce :

$$
\widehat{K}_{j m}=\int_{0}^{2 \pi}(\cos \varphi)^{j} \cos (|m| \varphi) d \varphi
$$

Converting cosines to exponentials and using again the Leibniz formula, it readily becomes :

$$
\widehat{K}_{j m}=\frac{1}{2^{j+1}} \sum_{k=0}^{j}\left(\begin{array}{l}
j \\
k
\end{array}\right) \int_{0}^{2 \pi}\left[e^{i(2 k-j-|m|) \varphi}+e^{i(2 k-j+|m|) \varphi}\right] d \varphi
$$


leading to :

$$
\widehat{K}_{j m}=\frac{2 \pi}{2^{j+1}}\left(\begin{array}{l}
j \\
k
\end{array}\right)\left[\delta_{0,2 k-j-|m|}+\delta_{0,2 k-j+|m|}\right]
$$

We can then deduce the following results :

(i) If $(j-m)$ is odd, or $j<|m|$, then :

$$
K_{j m}=\widehat{K}_{j m}=0
$$

(ii) If $(j-m)$ is even, and $|m| \leq j$,

then :

$$
K_{j m}=\widehat{K}_{j m}=\frac{2 \pi}{2^{j+1}}\left[\left(\begin{array}{c}
j \\
\frac{j+m}{2}
\end{array}\right)+\left(\begin{array}{c}
j \\
\frac{j-m}{2}
\end{array}\right)\right]
$$

\subsection{Corollary 2}

By using Eqs.10 and 11, we obtain the following fairly obvious corollary : if $n<|m|$, then :

$$
\int_{0}^{2 \pi} P_{n}(X(b)) e^{-i m(\varphi-b)} d \varphi=0
$$

We are now going to establish two identities to be used in the sequel.

\subsection{Lemma 3}

For any integers $r, s, n$ such that $s \leq r \leq[n / 2]$, in which [i] denotes the integer part of $i$, we have :

(i)

$$
\frac{1}{2^{n}} \sum_{m=0}^{s}(-1)^{m} \frac{2^{2 m}(2 n-2 m) !}{m !(n-m) !(s-m) !(r-m) !}=\frac{1}{r ! s !} \prod_{j=1}^{n-s}(2 j-1) \prod_{i=n-r-s+1}^{n-r}(2 i-1)
$$

with the convention that $\Pi_{i=i_{1}}^{i_{2}}=1$ if $i_{2}<i_{1}$, and :

(ii)

$$
\sum_{t=r}^{[n / 2]} \frac{1}{2^{2 t}(n-2 t) !(t-r) !(t-s) !}=\frac{(2(n-r-s)) !}{2^{n}(n-r-s) !(n-2 r) !(n-2 s) !}
$$

with the convention that $\sum_{i=i_{1}}^{i_{2}}=0$ if $i_{2}<i_{1}$.

\subsection{Proof of Lemma 3}

(i)

Let us denote by $S_{1}$ the 1.h.s. of Eq.13. But we have :

$$
\overline{S_{1}}=\frac{(2 n-2 m) !}{2^{n-m}(n-m) !}=\prod_{j=1}^{n-m}(2 j-1)
$$


Hence, $S_{1}$ can be rewritten as :

$$
\begin{aligned}
S_{1} & =\prod_{j=1}^{n-s}(2 j-1) \sum_{m=0}^{s}(-1)^{m} 2^{m} \frac{\prod_{j=n-s+1}^{n-m}(2 j-1)}{m !(s-m) !(r-m) !} \\
& =(-1)^{s} \frac{\prod_{j=1}^{n-s}(2 j-1)}{r ! s !} \sum_{m=0}^{s} 2^{m} m !\left(\begin{array}{c}
s \\
m
\end{array}\right)\left(\begin{array}{c}
r \\
m
\end{array}\right) \prod_{j=n-s+1}^{n-m}(1-2 j)
\end{aligned}
$$

From the r.h.s. of Eq.16, we introduce a polynomial $S_{1}(x)$ reading as :

$$
S_{1}(x)=\sum_{m=0}^{s} 2^{m} m !\left(\begin{array}{c}
s \\
m
\end{array}\right)\left(\begin{array}{c}
r \\
m
\end{array}\right) \prod_{j=n-s+1}^{n-m}(x+1-2 j)
$$

We observe that $S_{1}(x)$ is a polynomial of degree $s$ written in the Newton basis $\left\{N_{i}(x)\right\}_{i=0}^{s}$ with $N_{0}(x)=1$ and $N_{i+1}(x)=\left(x-x_{i}\right) N_{i}(x)$ for $i \geqslant 0$, where $x_{0}=2 n-2 s+1, x_{k}=x_{0}+k h$ and $h=2$. Thus :

$$
\prod_{j=n-s+1}^{n-m}(x+1-2 j)=N_{s-m}(x)
$$

Let us prove that :

$$
S_{1}(x)=Q(x)=\prod_{i=n-r-s+1}^{n-r}(x-2 i+1)
$$

It is sufficient to verify that $S_{1}\left(x_{k}\right)=Q\left(x_{k}\right)$ for $\mathrm{k}=0 \ldots \mathrm{s}$. We have :

$$
Q\left(x_{k}\right)=2^{s} \frac{(r+k) !}{(r+k-s) !}
$$

Since :

$$
S_{1}(x)=\sum_{m=0}^{s} 2^{s-m} \frac{s !}{m !}\left(\begin{array}{c}
r \\
s-m
\end{array}\right) N_{m}(x)
$$

and :

$$
N_{m}\left(x_{k}\right)=2^{m} \frac{k !}{(k-m) !}
$$

we obtain :

$$
S_{1}\left(x_{k}\right)=2^{s} \frac{k ! r !}{(r-s+k) !} \sum_{m=0}^{k}\left(\begin{array}{c}
s \\
m
\end{array}\right)\left(\begin{array}{c}
r-s+k \\
k-m
\end{array}\right)
$$

in which the summation is originally found to range from $m=0$ to $s$, but can afterward be reduced from $m=0$ to $k$. By using the following identity (Abramowitz \& Stegun (1964), p822)

$$
\sum_{m=0}^{n}\left(\begin{array}{c}
r \\
m
\end{array}\right)\left(\begin{array}{c}
s \\
n-m
\end{array}\right)=\left(\begin{array}{c}
r+s \\
n
\end{array}\right), \forall(r+s) \geq n
$$

we have :

$$
S_{1}\left(x_{k}\right)=\frac{2^{s} k ! r !}{(r-s+k) !}\left(\begin{array}{c}
r+k \\
k
\end{array}\right)=\frac{2^{s}(r+k) !}{(r+k-s) !}=Q\left(x_{k}\right)
$$


But :

$$
S_{1}=\frac{(-1)^{s}}{r ! s !} \prod_{j=1}^{n-s}(2 j-1) S_{1}(0)
$$

Hence, it is readily seen that the first identity holds.

(ii)

On one hand, we introduce the quantity $B_{2}$ according to :

$$
\begin{aligned}
(n-2 r) ! B_{2} & =\frac{1}{2^{r+s}(n-2 s) !} \prod_{i=1}^{n-r-s}(2 i-1) \\
& =\frac{1}{2^{r+s}(n-2 s) !} \prod_{i=1}^{n-[n / 2]-s}(2 i-1) \prod_{i=n-[n / 2]-s+1}^{n-r-s}(2 i-1)
\end{aligned}
$$

Next, let $R(x)$ denote the polynomial of degree $[n / 2]-r$, given by :

$$
R(x)=\frac{1}{2^{r+s}(n-2 s) !} \prod_{i=1}^{n-[n / 2]-s}(2 i-1) \prod_{i=n-[n / 2]-s+1}^{n-r-s}(x+2 i-1)
$$

so that :

$$
(n-2 r) ! B_{2}=R(0)
$$

On the other hand, we have :

$$
(n-2 r) ! S_{2}=\sum_{t=r}^{[n / 2]} \frac{1}{2^{2 t}} \frac{\prod_{j=r}^{t-1}(n-2 j) \prod_{j=r}^{t-1}(n-2 j-1)}{(t-r) !(t-s) !}
$$

One of the two products only involves even integers while the other only involves odd integers. Thus, we can write them as :

$$
\prod_{j=r}^{t-1} 2([n / 2]-j) \prod_{j=r}^{t-1}(2 n-2[n / 2]-2 j-1)
$$

Therefore, with fairly obvious changes of variables :

$$
(n-2 r) ! S_{2}=\sum_{t=0}^{[n / 2]-r} \frac{1}{2^{2 t+2 r} t !(t+r-s) !} \prod_{j=0}^{t-1} 2([n / 2]-j-r) \prod_{j=0}^{t-1}[2(n-[n / 2]-j-r)-1]
$$

Let $\widetilde{S_{2}}(x)$ denote the polynomial :

$$
\widetilde{S_{2}}(x)=\sum_{t=0}^{[n / 2]-r} \frac{1}{2^{2 t+2 r} t !(t+r-s) !} \prod_{j=0}^{t-1} 2([n / 2]-j-r) \prod_{j=0}^{t-1}[x+2(n-[n / 2]-j-r)-1]
$$

so that:

$$
(n-2 r) ! S_{2}=\widetilde{S_{2}}(0)
$$


The polynomial $\widetilde{S_{2}}(x)$, which is of degree $([n / 2]-r)$, is written in the Newton basis $\left\{N_{m}(x)\right\}_{m=0}^{[n / 2]-r}$, with $x_{0}=1-2(n-[n / 2]-r)$ and $x_{m}=x_{0}+m h, m=0 \ldots[n / 2]-r, h=2$.

Let us now prove that $\widetilde{S_{2}}(x)$ and $R(x)$ are identical, which is equivalent to verify that $\widetilde{S_{2}}\left(x_{m}\right)=$ $R\left(x_{m}\right), m=0 \ldots[n / 2]-r$.

We have :

$$
\begin{aligned}
\widetilde{S_{2}}\left(x_{m}\right) & =\sum_{t=0}^{m} \frac{1}{2^{2 t+2 r}} \frac{\prod_{j=0}^{t-1} 2([n / 2]-j-r)}{t !(t+r-s) !} \frac{2^{t} m !}{(m-t) !} \\
& =\frac{1}{2^{2 r}} \sum_{t=0}^{m} \frac{([n / 2]-r) ! m !}{t !(t+r-s) !([n / 2]-r-t) !(m-t) !} \\
& =\frac{m !}{2^{2 r}(m+r-s) !} \sum_{t=0}^{m}\left(\begin{array}{c}
{[n / 2]-r} \\
t
\end{array}\right)\left(\begin{array}{c}
m+r-s \\
m-t
\end{array}\right)
\end{aligned}
$$

By using Eq.24, this becomes :

$$
\widetilde{S_{2}}\left(x_{m}\right)=\frac{m !}{2^{2 r}(m+r-s) !}\left(\begin{array}{c}
{[n / 2]+m-s} \\
m
\end{array}\right)=\frac{([n / 2]+m-s) !}{2^{2 r}(n+r-s) !([n / 2]-s) !}
$$

Next, we have :

$$
\begin{aligned}
R\left(x_{m}\right) & =\frac{1}{2^{r+s}(n-2 s) !} \prod_{i=1}^{n-[n / 2]-s}(2 i-1) \prod_{i=1}^{[n / 2]-r} 2(r+m+i-s) \\
& =\frac{1}{2^{2 r+n-2[n / 2]}} \frac{(2(n-[n / 2]-s)) !([n / 2]+m-s) !}{(n-2 s) !(n-[n / 2]-s) !(r+m-s) !}
\end{aligned}
$$

For any integer $n$, even or odd, we readily have :

$$
(2(n-[n / 2]-s)) !([n / 2]-s) !=2^{n-2[n / 2]}(n-2 s) !(n-[n / 2]-s) !
$$

Therefore :

$$
R\left(x_{m}\right)=\widetilde{S_{2}}\left(x_{m}\right), m=0 \ldots[n / 2]-r
$$

Hence, because :

$$
S_{2}=\frac{\widetilde{S_{2}}(0)}{(n-2 r) !}=\frac{R(0)}{(n-2 r) !}
$$

the second identity holds.

\subsection{Theorem 4}

We can now prove a first theorem reading as :

$$
\int_{0}^{2 \pi} P_{n}(\sin a \sin \theta \cos \varphi+\cos a \cos \theta) d \varphi=2 \pi P_{n}(\cos a) P_{n}(\cos \theta)
$$

where $P_{n}$ is the Legendre polynomial of degree $n$. 


\subsection{Proof of Theorem 4}

By using an expansion of Legendre polynomials (e.g. G.B.Arfken \& H.J.Weber (2005), p744), we obtain :

$$
\begin{aligned}
& P_{n}(\sin a \sin \theta \cos \varphi+\cos a \cos \theta) \\
= & \frac{1}{2^{n}} \sum_{m=0}^{[n / 2]}(-1)^{m}\left(\begin{array}{c}
n \\
m
\end{array}\right)\left(\begin{array}{c}
2 n-2 m \\
n
\end{array}\right)(\sin a \sin \theta \cos \varphi+\cos a \cos \theta)^{n-2 m}
\end{aligned}
$$

But we have :

$$
\begin{aligned}
& \int_{0}^{2 \pi}(\sin a \sin \theta \cos \varphi+\cos a \cos \theta)^{n-2 m} d \varphi \\
= & \sum_{j=0}^{n-2 m}\left(\begin{array}{c}
n-2 m \\
j
\end{array}\right) \int_{0}^{2 \pi}(\sin a \sin \theta \cos \varphi)^{j}(\cos a \cos \theta)^{n-2 m-j} d \varphi \\
= & \sum_{k=0}^{\left.\frac{n-2 m}{2}\right]}\left(\begin{array}{c}
n-2 m \\
2 k
\end{array}\right) \int_{0}^{2 \pi}(\sin a \sin \theta \cos \varphi)^{2 k}(\cos a \cos \theta)^{n-2 m-2 k} d \varphi
\end{aligned}
$$

in which we have used (see Eq.6) :

$$
\int_{0}^{2 \pi}(\cos \varphi)^{j} d \varphi=0, \text { for any odd integer } j
$$

Using again Eq.6 for $j$ even, we obtain :

$$
\begin{aligned}
& \int_{0}^{2 \pi}(\sin a \sin \theta \cos \varphi+\cos a \cos \theta)^{n-2 m} d \varphi \\
= & \sum_{k=0}^{\left[\frac{n-2 m}{2}\right]} \frac{2 \pi}{2^{2 k}}\left(\begin{array}{c}
n-2 m \\
2 k
\end{array}\right)\left(\begin{array}{c}
2 k \\
k
\end{array}\right)\left(1-\cos ^{2} a\right)^{k}\left(1-\cos ^{2} \theta\right)^{k}(\cos a \cos \theta)^{n-2 m-2 k}
\end{aligned}
$$

Eq.45 implies that $\int_{0}^{2 \pi} P_{n}(\sin a \sin \theta \cos \varphi+\cos a \cos \theta) d \varphi$ is a polynomial $q_{n}(\cos a, \cos \theta)$, symmetrical with respect to the two variables $\cos a$ and $\cos \theta$, of degree $n$ with respect to each variable. We then invoke the Leibniz formula to develop $\left(1-\cos ^{2} a\right)^{k}$ and $\left(1-\cos ^{2} \theta\right)^{k}$ involved in Eq.45 leading to :

$$
\begin{aligned}
q_{n}(\cos a, \cos \theta)= & \frac{2 \pi}{2^{n}} \sum_{m=0}^{[n / 2]} \sum_{k=0}^{\left[\frac{n-2 m}{2}\right]} \sum_{j=0}^{k} \sum_{i=0}^{k} \frac{(-1)^{m-j-i}}{2^{2 k}}\left(\begin{array}{c}
n \\
m
\end{array}\right)\left(\begin{array}{c}
2 n-2 m \\
n
\end{array}\right) \\
& \left(\begin{array}{c}
n-2 m \\
2 k
\end{array}\right)\left(\begin{array}{c}
2 k \\
k
\end{array}\right)\left(\begin{array}{c}
k \\
k-j
\end{array}\right)\left(\begin{array}{c}
k \\
k-i
\end{array}\right)(\cos a)^{n-2 m-2 i}(\cos \theta)^{n-2 m-2 j}
\end{aligned}
$$

We now intend to identify Eq.46 and the expansion of $2 \pi P_{n}(\cos a) P_{n}(\cos \theta)$ with respect to the variables $\cos a$ and $\cos \theta$. 
For a fixed integer $r$ such that $r=m+j$ and $r \leq[n / 2]$, and a fixed integer $s$ such that $s=m+i$ and $s \leq[n / 2]$, the factor of $(\cos a)^{n-2 s}(\cos \theta)^{n-2 r}$ in Eq.46 can be obtained and compared with its counterpart in $2 \pi P_{n}(\cos a) P_{n}(\cos \theta)$. This counterpart reads as :

$$
\frac{2 \pi}{2^{2 n}}(-1)^{r+s}\left(\begin{array}{l}
n \\
r
\end{array}\right)\left(\begin{array}{l}
n \\
s
\end{array}\right)\left(\begin{array}{c}
2 n-2 r \\
n
\end{array}\right)\left(\begin{array}{c}
2 n-2 s \\
n
\end{array}\right)
$$

Since $q_{n}$ is a symmetrical polynomial, it is sufficient to carry out the comparison for $s \leq r$. To approach the aim, we interchange $k$ - and $j-$ summations in Eq.46, according to :

$$
\sum_{k=0}^{\left[\frac{n-2 m}{2}\right]} \sum_{j=0}^{k}=\sum_{j=0}^{\left[\frac{n-2 m}{2}\right]} \sum_{k=j}^{\left[\frac{n-2 m}{2}\right]}
$$

Therefore, for $m+j=r$, the factor of $(\cos \theta)^{n-2 r}$ is :

$$
\begin{aligned}
& \frac{2 \pi}{2^{n}} \sum_{m=0}^{r} \sum_{k=r-m}^{\left[\frac{n-2 m}{2}\right]} \sum_{i=0}^{k} \\
& \frac{(-1)^{r-i}}{2^{2 k}}\left(\begin{array}{c}
n \\
m
\end{array}\right)\left(\begin{array}{c}
2 n-2 m \\
n
\end{array}\right)\left(\begin{array}{c}
n-2 m \\
2 k
\end{array}\right)\left(\begin{array}{c}
2 k \\
k
\end{array}\right)\left(\begin{array}{c}
k \\
m+k-r
\end{array}\right)\left(\begin{array}{c}
k \\
k-i
\end{array}\right) \\
& (\cos a)^{n-2 m-2 i}
\end{aligned}
$$

Next, we interchange $k$ - and $i$-summations, according to :

$$
\sum_{k=r-m}^{\left[\frac{n-2 m}{2}\right]} \sum_{i=0}^{k}=\sum_{i=0}^{r-m} \sum_{k=r-m}^{\left[\frac{n-2 m}{2}\right]}+\sum_{i=r-m+1}^{\left[\frac{n-2 m}{2}\right]} \sum_{k=i}^{\left[\frac{n-2 m}{2}\right]}
$$

Thus, for $m+i=s \leq r$, the factor of $(\cos a)^{n-2 s}(\cos \theta)^{n-2 r}$ is :

$$
\begin{aligned}
& \frac{2 \pi}{2^{n}} \sum_{m=0}^{s} \sum_{k=r-m}^{\left[\frac{n-2 m}{2}\right]} \frac{(-1)^{m+r+s}}{2^{2 k}}\left(\begin{array}{c}
n \\
m
\end{array}\right)\left(\begin{array}{c}
2 n-2 m \\
n
\end{array}\right)\left(\begin{array}{c}
n-2 m \\
2 k
\end{array}\right) \\
& \left(\begin{array}{c}
2 k \\
k
\end{array}\right)\left(\begin{array}{c}
k \\
m+k-r
\end{array}\right)\left(\begin{array}{c}
k \\
m+k-s
\end{array}\right)
\end{aligned}
$$

which is equal to :

$$
\begin{aligned}
& (-1)^{r+s} \frac{2 \pi}{2^{n}} \sum_{m=0}^{s}(-1)^{m} \frac{(2 n-2 m) !}{m !(n-m) !(s-m) !(r-m) !} \\
& \sum_{k=r-m}^{[n / 2]-m} \frac{1}{2^{2 k}(n-2 m-2 k) !(m+k-r) !(m+k-s) !}
\end{aligned}
$$

By using the two identities 13 and 14 from Lemma 3, this result may be rewritten as :

$$
(-1)^{r+s} \frac{2 \pi}{r ! s !} \prod_{j=1}^{n-s}(2 j-1) \prod_{i=n-r-s+1}^{n-r}(2 i-1) \frac{(2(n-r-s)) !}{2^{n}(n-r-s) !(n-2 r) !(n-2 s) !}
$$


which is equal to the expression 47 . Hence, the proof is done.

\subsection{Corollary 5. Reproducing kernel}

A new expression of the reproducing kernel of Legendre polynomials can be readily derived from Theorem 4 . We then obtain a Corollary 5 as follows.

The reproducing kernel $K_{n}(x, t)$ of Legendre polynomials $P_{i}(x)$ :

$$
K_{n}(x, t)=\sum_{i=0}^{n} \frac{2 i+1}{2} P_{i}(x) P_{i}(t)
$$

has the following integral representation :

$$
K_{n}(x, t)=\frac{1}{2 \pi} \int_{0}^{2 \pi} \sum_{i=0}^{n} \frac{2 i+1}{2} P_{i}\left(x t+\cos \varphi \sqrt{1-x^{2}} \sqrt{\left.1-t^{2}\right)} d \varphi\right.
$$

\subsection{Jacobi polynomials}

For further use, we now recall some results concerning Jacobi polynomials $P_{n}^{(\alpha, \beta)}(x), \alpha>-1$ and $\beta>-1$ Abramowitz \& Stegun (1964), T.S.Chihara (1978), Szegö (1939).

Jacobi polynomials are orthogonal with respect to the linear functional :

$$
\int_{-1}^{+1} \cdot(1-x)^{\alpha}(1+x)^{\beta} d x, \alpha, \beta>-1
$$

They have the following $L^{2}-$ norm :

$$
\int_{-1}^{+1}(1-x)^{\alpha}(1+x)^{\beta}\left[P_{n}^{(\alpha, \beta)}(x)\right]^{2} d x=\frac{2^{\alpha+\beta+1}}{2 n+\alpha+\beta+1} \frac{\Gamma(n+\alpha+1) \Gamma(n+\beta+1)}{\Gamma(n+1) \Gamma(n+\alpha+\beta+1)}
$$

where $\Gamma$ is the Gamma function.

The derivative of $P_{n}^{(\alpha, \beta)}(x)$ is another Jacobi polynomial :

$$
\frac{d}{d x} P_{n}^{(\alpha, \beta)}(x)=\frac{1}{2}(n+\alpha+\beta+1) P_{n-1}^{(\alpha+1, \beta+1)}(x)
$$

We now provide relations valid for $\alpha=\beta>-1$.

Jacobi polynomials, for $\alpha=\beta>-1$, satisfy a three-term recurrence relation :

$$
\begin{aligned}
& (n+\alpha+1)(2 n+2 \alpha+1) x P_{n}^{(\alpha, \alpha)}(x) \\
= & (n+1)(n+2 \alpha+1) P_{n+1}^{(\alpha, \alpha)}(x)+(n+\alpha)(n+\alpha+1) P_{n-1}^{(\alpha, \alpha)}(x)
\end{aligned}
$$

with $P_{0}^{(\alpha, \alpha)}=1$ and $P_{-1}^{(\alpha, \alpha)}=0$. 
Furthermore :

$$
\begin{aligned}
& (2 n+2 \alpha-1) P_{n}^{(\alpha-1, \alpha-1)}(x) \\
= & \frac{(n+2 \alpha-1)(n+2 \alpha)}{2(n+\alpha)} P_{n}^{(\alpha, \alpha)}(x)-\frac{(n+\alpha-1)}{2} P_{n-2}^{(\alpha, \alpha)}(x) \\
& \frac{2 n+2 \alpha+3}{2}\left(1-x^{2}\right) P_{n}^{(\alpha+1, \alpha+1)}(x) \\
= & (n+\alpha+1) P_{n}^{(\alpha, \alpha)}(x)-\frac{(n+1)(n+2)}{n+\alpha+2} P_{n+2}^{(\alpha, \alpha)}(x) \\
\left(1-x^{2}\right) \frac{d}{d x} P_{n}^{(\alpha, \alpha)}(x)= & \left(1-x^{2}\right) \frac{n+2 \alpha+1}{2} P_{n-1}^{(\alpha+1, \alpha+1)}(x) \\
= & -n x P_{n}^{(\alpha, \alpha)}(x)+(n+\alpha) P_{n-1}^{(\alpha, \alpha)}(x)
\end{aligned}
$$

We now know enough to prove the main theorem.

\subsection{Theorem 6}

For any integer $m \in \mathbf{Z}$ and any integer $n \in \mathbf{N}$ :

$$
\begin{aligned}
& \int_{0}^{2 \pi} P_{n}(\sin a \sin \theta \cos (\varphi-b)+\cos a \cos \theta) e^{-i m(\varphi-b)} d \varphi \\
= & 2 \pi \frac{(n-|m|) !}{(n+|m|) !} P_{n}^{|m|}(\cos a) P_{n}^{|m|}(\cos \theta)
\end{aligned}
$$

where $i=\sqrt{-1}, P_{n}$ is the Legendre polynomial of degree $n$, and $P_{n}^{|m|}$ is the associated Legendre function of order $|m|$ defined by:

$$
P_{n}^{|m|}(x)=(-1)^{|m|}\left(1-x^{2}\right)^{\frac{|m|}{2}} \frac{d^{|m|}}{d x^{|m|}} P_{n}(x)
$$

\subsection{Proof of Theorem 6}

We conveniently introduce, for further use, a specific notation for the 1.h.s. of Eq.63 :

$$
D_{n m}=\int_{0}^{2 \pi} P_{n}(\sin a \sin \theta \cos (\varphi-b)+\cos a \cos \theta) e^{-i m(\varphi-b)} d \varphi
$$

Now, it happens that Eq.63 of Theorem 6 is already proved for $m=0$ (Theorem 4) and for $n<|m|$ (Corollary 2). Moreover, by using Lemma 1, Eq.63 is equivalent to :

$$
\int_{0}^{2 \pi} P_{n}(\sin a \sin \theta \cos \varphi+\cos a \cos \theta) \cos (|m| \varphi) d \varphi=2 \pi \frac{(n-|m|) !}{(n+|m|) !} P_{n}^{|m|}(\cos a) P_{n}^{|m|}(\cos \theta)
$$


But $P_{j}^{|m|}(x)$ possesses an expression in terms of the Jacobi polynomial $P_{j-|m|}^{(|m|,|m|)}(x)$. Indeed, by using Eqs.58 and 64 :

$$
P_{j}^{|m|}(x)=(-1)^{|m|}\left(1-x^{2}\right)^{\frac{|m|}{2}} \frac{(j+1)_{|m|}}{2^{|m|}} P_{j-|m|}^{(|m|,|m|)}(x)
$$

where $(c)_{j}$ is the Pochhammer symbol :

$$
\left.\begin{array}{c}
(c)_{j}=c(c+1) \ldots(c+j-1), \forall j \in \mathbf{N} \\
(c)_{0}=1
\end{array}\right\}
$$

We now prove Eq.63 for $m=1$. From Eq.59 with $\alpha=0$ and Eq.67, we have :

$$
\begin{aligned}
& (2 n+1) X P_{n}(X) \\
= & (2 n+1) \sin a \sin \theta \cos \varphi P_{n}(X)+(2 n+1) \cos a \cos \theta P_{n}(X) \\
= & (n+1) P_{n+1}(X)+n P_{n-1}(X)
\end{aligned}
$$

in which we conveniently used $X=X(b=0)$. Hence, by using Theorem 4 , and remembering Lemma 1 and its associated remark :

$$
\begin{aligned}
& (2 n+1) \sin a \sin \theta D_{n 1} \\
= & (n+1) D_{n+1,0}-(2 n+1) \cos a \cos \theta D_{n 0}+n D_{n-1,0} \\
= & 2 \pi(n+1) P_{n+1}(\cos a) P_{n+1}(\cos \theta)-2 \pi(2 n+1) \cos a \cos \theta P_{n}(\cos a) P_{n}(\cos \theta) \\
& +2 \pi n P_{n-1}(\cos a) P_{n-1}(\cos \theta)
\end{aligned}
$$

With Eq.59 for $\alpha=0$, this expression becomes :

$$
\begin{aligned}
& \frac{2 \pi}{n+1}\left[(2 n+1) \cos a P_{n}(\cos a)-n P_{n-1} \cos (a)\right] \\
& {\left[(2 n+1) \cos \theta P_{n}(\cos \theta)-n P_{n-1}(\cos \theta)\right]} \\
& -2 \pi(2 n+1) \cos a \cos \theta P_{n}(\cos a) P_{n}(\cos \theta)+2 \pi n P_{n-1}(\cos a) P_{n-1}(\cos \theta)
\end{aligned}
$$

which can be factorized to :

$$
2 \pi \frac{(2 n+1)}{n(n+1)}\left[-n \cos a P_{n}(\cos a)+n P_{n-1}(\cos a)\right]\left[-n \cos \theta P_{n}(\cos \theta)+n P_{n-1}(\cos \theta)\right]
$$

This expression is identical to :

$$
2 \pi(2 n+1) \sin a \sin \theta \frac{(n-1) !}{(n+1) !} P_{n}^{1}(\cos a) P_{n}^{1}(\cos \theta)
$$

This result is obtained by using Eq.62 with $\alpha=0$ and Eq.64 for $m=1$, namely :

$$
\begin{gathered}
(\sin a)^{2} \frac{d}{d \cos a} P_{n}(\cos a)=-n \cos a P_{n}(\cos a)+n P_{n-1}(\cos a) \\
P_{n}^{1}(\cos a)=-\sin a \frac{d}{d \cos a} P_{n}(\cos a)
\end{gathered}
$$


Therefore, Eq.63 holds for $m=1$. Now, we can complete the demonstration of Eq.63 by recurrence, assuming that it is satisfied by any integer $n \in \mathbf{N}$ up to $(m-1) \geq 1$ (we can assume that $m$ is positive).

We have :

$$
\cos (m \varphi)=2 \cos \varphi \cos ((m-1) \varphi)-\cos ((m-2) \varphi)
$$

From Eqs.76 and 69, we obtain :

$$
\begin{aligned}
& \sin a \sin \theta P_{n}(X) \cos (m \varphi) \\
= & \frac{2}{2 n+1} \cos ((m-1) \varphi)\left[(n+1) P_{n+1}(X)-(2 n+1) \cos a \cos \theta P_{n}(X)+n P_{n-1}(X)\right] \\
& -\sin a \sin \theta P_{n}(X) \cos ((m-2) \varphi)
\end{aligned}
$$

Hence :

$$
\begin{aligned}
& \sin a \sin \theta D_{n m}=\frac{2}{2 n+1}\left[(n+1) D_{n+1, m-1}-(2 n+1) \cos a \cos \theta D_{n, m-1}+n D_{n-1, m-1}\right] \\
& -\sin a \sin \theta D_{n, m-2}
\end{aligned}
$$

We now use the recurrence assumption, yielding, from Theorem 6 :

$$
\begin{aligned}
\sin a \sin \theta D_{n m}= & \frac{4 \pi}{2 n+1}\left[(n+1) \frac{(n-m+2) !}{(n+m) !} P_{n+1}^{m-1}(\cos a) P_{n+1}^{m-1}(\cos \theta)\right. \\
& -(2 n+1) \frac{(n-m+1) !}{(n+m-1) !} \cos a \cos \theta P_{n}^{m-1}(\cos a) P_{n}^{m-1}(\cos \theta) \\
& \left.+n \frac{(n-m) !}{(n+m-2) !} P_{n-1}^{m-1}(\cos a) P_{n-1}^{m-1}(\cos \theta)\right] \\
& -2 \pi \frac{(n-m+2) !}{(n+m-2) !} \sin a \sin \theta P_{n}^{m-2}(\cos a) P_{n}^{m-2}(\cos \theta)
\end{aligned}
$$

Every $P_{j}^{m-1}$ and $P_{j}^{m-2}$ is replaced by using the expression 67 , leading to :

$$
\begin{aligned}
\sin a \sin \theta D_{n m}= & 2 \pi \frac{(n-m) !}{(n+m) !} \frac{(\sin a \sin \theta)^{m-1}}{2^{2 m-4}}\left((n+1)_{m}\right)^{2}\left\{\frac{1}{2(2 n+1)}\right. \\
& {\left[\frac{(n-m+1)_{2}}{n+1} P_{n-m+2}^{(m-1, m-1)}(\cos a) P_{n-m+2}^{(m-1, m-1)}(\cos \theta)\right.} \\
& -(2 n+1) \frac{n-m+1}{n+m} \cos a \cos \theta P_{n-m+1}^{(m-1, m-1)}(\cos a) P_{n-m+1}^{(m-1, m-1)}(\cos \theta) \\
& \left.+\frac{n^{3}}{(n+m-1)_{2}} P_{n-m}^{(m-1, m-1)}(\cos a) P_{n-m}^{(m-1, m-1)}(\cos \theta)\right] \\
& \left.-\frac{(n-m+1)_{2}}{(n+m-1)_{2}} P_{n-m+2}^{(m-2, m-2)}(\cos a) P_{n-m+2}^{(m-2, m-2)}(\cos \theta)\right\}
\end{aligned}
$$

We afterward substitute $P_{n-m+2}^{(m-2, m-2)}$ for $P_{j}^{(m-1, m-1)}$ by using Eq.60 and $\cos a P_{n-m+1}^{(m-1, m-1)}(\cos a)$ for $P_{j}^{(m-1, m-1)}$ by using Eq.59. We then obtain : 


$$
\begin{aligned}
\sin a \sin \theta D_{n m}= & 2 \pi \frac{(n-m) !}{(n+m) !} \frac{(\sin a \sin \theta)^{m-1}}{2^{2 m-4}}((n+1))^{2}\left\{\frac{1}{2(2 n+1)}\right. \\
& {\left[\frac{(n-m+1)_{2}}{n+1} P_{n-m+2}^{(m-1, m-1)}(\cos a) P_{n-m+2}^{(m-1, m-1)}(\cos \theta)\right.} \\
& -\frac{n-m+1}{(n+m)(n+1)^{2}(2 n+1)}\left((n+m)(n-m+2) P_{n-m+2}^{(m-1, m-1)}(\cos a)\right. \\
& \left.+n(n+1) P_{n-m}^{(m-1, m-1)}(\cos a)\right)\left((n+m)(n-m+2) P_{n-m+2}^{(m-1, m-1)}(\cos \theta)\right. \\
& \left.+n(n+1) P_{n-m}^{(m-1, m-1)}(\cos \theta)\right)+ \\
& \left.\frac{n^{3}}{(n+m-1)_{2}} P_{n-m}^{(m-1, m-1)}(\cos a) P_{n-m}^{(m-1, m-1)}(\cos \theta)\right] \\
& -\frac{(n-m+1)_{2}}{(2 n+1)^{2}(n+m-1)_{2}}\left(\frac{(n+m-1)_{2}}{2(n+1)} P_{n-m+2}^{(m-1, m-1)}(\cos a)\right. \\
& \left.-\frac{n}{2} P_{n-m}^{(m-1, m-1)}(\cos a)\right) \\
& \left.\left(\frac{(n+m-1)_{2}}{2(n+1)} P_{n-m+2}^{(m-1, m-1)}(\cos \theta)-\frac{n}{2} P_{n-m}^{(m-1, m-1)}(\cos \theta)\right)\right\}
\end{aligned}
$$

becoming :

$$
\begin{aligned}
\sin a \sin \theta D_{n m}= & 2 \pi \frac{(n-m) !}{(n+m) !} \frac{(\sin a \sin \theta)^{m-1}}{2^{2 m-4}}\left((n+1)_{m}\right)^{2} \\
& {\left[\frac{\left((n-m+1)_{2}\right)^{2}}{4(n+1)^{2}(2 n+1)^{2}} P_{n-m+2}^{(m-1, m-1)}(\cos a) P_{n-m+2}^{(m-1, m-1)}(\cos \theta)\right.} \\
& -\frac{n(n-m+1)_{2}}{4(n+1)(2 n+1)^{2}}\left(P_{n-m+2}^{(m-1, m-1)}(\cos a) P_{n-m}^{(m-1, m-1)}(\cos \theta)\right. \\
& \left.+P_{n-m+2}^{(m-1, m-1)}(\cos \theta) P_{n-m}^{(m-1, m-1)}(\cos a)\right) \\
& \left.+\frac{n^{2}}{4(2 n+1)^{2}} P_{n-m}^{(m-1, m-1)}(\cos a) P_{n-m}^{(m-1, m-1)}(\cos \theta)\right]
\end{aligned}
$$

which factorizes to :

$$
\begin{aligned}
\sin a \sin \theta D_{n m}= & 2 \pi \frac{(n-m) !}{(n+m) !} \frac{(\sin a \sin \theta)^{m-1}}{2^{2 m-2}} \frac{\left((n+1)_{m}\right)^{2}}{(2 n+1)^{2}} \\
& {\left[n P_{n-m}^{(m-1, m-1)}(\cos a)-\frac{(n-m+1)_{2}}{n+1} P_{n-m+2}^{(m-1, m-1)}(\cos a)\right] } \\
& {\left[n P_{n-m}^{(m-1, m-1)}(\cos \theta)-\frac{(n-m+1)_{2}}{n+1} P_{n-m+2}^{(m-1, m-1)}(\cos \theta)\right] }
\end{aligned}
$$

We then invoke Eq.61, with $\alpha=m-1, n \mapsto n-m$, to obtain :

$$
\sin a \sin \theta D_{n m}=2 \pi \frac{(n-m) !}{(n+m) !} \frac{(\sin a \sin \theta)^{m+1}}{2^{2 m}}((n+1) m)^{2} P_{n-m}^{(m, m)}(\cos a) P_{n-m}^{(m, m)}(\cos \theta)
$$


Therefore, recalling the definition of $D_{n m}$, we find that Eq.63, i.e. Theorem 6, holds for any integer $m \in \mathbf{Z}$.

\subsection{A consequence}

An important consequence of Theorem 6 concerns the spherical harmonics $Y_{j}^{m}(\theta, \varphi)$ which are defined as :

$$
Y_{j}^{m}(\theta, \varphi)=\sqrt{\frac{(2 j+1)}{4 \pi} \frac{(j-m) !}{(j+m) !}} P_{j}^{m}(\cos \theta) e^{i m \varphi}
$$

When $m<0, P_{j}^{m}(\cos \theta)$ is defined as :

$$
P_{j}^{m}(\cos \theta)=(-1)^{m} \frac{(j+m) !}{(j-m) !} P_{j}^{-m}(\cos \theta)
$$

Therefore, we may uniquely define $P_{j}^{m}(\cos \theta)$ for any integer $m \in \mathbf{Z}$, according to :

$$
P_{j}^{m}(\cos \theta)=(-1)^{\frac{m-|m|}{2}} \frac{(j-|m|) !}{(j-m) !} P_{j}^{|m|}(\cos \theta)
$$

in which $P_{j}^{|m|}(x)$ is defined by Eq.64.

Eq.85 can then be given an unique form for any integer $m \in \mathbf{Z}$, reading as :

$$
Y_{j}^{m}(\theta, \varphi)=(-1)^{\frac{m-|m|}{2}} \sqrt{\frac{2 j+1}{4 \pi}} \sqrt{\frac{(j-m) !}{(j+m) !}} \frac{(j-|m|) !}{(j-m) !} P_{j}^{|m|}(\cos \theta) e^{i m \varphi}
$$

simplifying to :

$$
Y_{j}^{m}(\theta, \varphi)=(-1)^{\frac{m-|m|}{2}} \sqrt{\frac{2 j+1}{4 \pi}} \sqrt{\frac{(j-|m|) !}{(j+|m|) !}} P_{j}^{|m|}(\cos \theta) e^{i m \varphi}
$$

From these equations, the complex conjuguate of $Y_{j}^{m}(\theta, \varphi)$ is :

$$
\overline{Y_{j}^{m}(\theta, \varphi)}=(-1)^{m} Y_{j}^{-m}(\theta, \varphi), \forall m \in \mathbf{Z}
$$

\subsection{Corollary 7}

For any integers $n, j \in \mathbf{N}$, and any integer $m \in \mathbf{Z}$ :

$$
\begin{aligned}
& \int_{0}^{\pi}\left[\int_{0}^{2 \pi} P_{n}(\sin a \sin \theta \cos (\varphi-b)+\cos a \cos \theta) e^{-i m(\varphi-b)} d \varphi\right] \\
& P_{j}^{m}(\cos \theta) \sin \theta d \theta \\
= & \frac{4 \pi}{2 j+1} P_{j}^{m}(\cos a) \delta_{n j}
\end{aligned}
$$




\subsection{Proof of Corollary 7}

>From Theorem 6 and Eq.87, the 1.h.s. of Eq.91 is :

$$
L H S=2 \pi(-1)^{\frac{m-|m|}{2}} \frac{(n-|m|) !}{(n+|m|) !} \frac{(j-|m|) !}{(j-m) !} P_{n}^{|m|}(\cos a) \int_{0}^{\pi} P_{n}^{|m|}(\cos \theta) P_{j}^{|m|}(\cos \theta) \sin \theta d \theta
$$

But, using Eqs.67 and 57 :

$$
\begin{aligned}
& \int_{0}^{\pi} P_{n}^{|m|}(\cos \theta) P_{j}^{|m|}(\cos \theta) \sin \theta d \theta \\
= & \frac{1}{2^{2|m|}}(n+1)_{|m|}(j+1)_{|m|} \int_{-1}^{+1} P_{n-|m|}^{(|m|,|m|)}(x) P_{j-|m|}^{(|m|,|m|)}(x)\left(1-x^{2}\right)^{|m|} d x \\
= & \frac{1}{2^{2|m|}}((n+1)|m|)^{2} \frac{2^{2|m|+1}}{2 n+1} \frac{(\Gamma(n+1))^{2}}{\Gamma(n-|m|+1) \Gamma(n+|m|+1)} \delta_{n j}=\frac{2}{2 n+1} \frac{(n+|m|) !}{(n-|m|) !} \delta_{n j}
\end{aligned}
$$

Therefore, Eq.92 becomes :

$$
\text { LHS }=\frac{4 \pi}{2 n+1}(-1)^{\frac{m-|m|}{2}} \frac{(n-|m|) !}{(n-m) !} P_{n}^{|m|}(\cos a) \delta_{n j}=\frac{4 \pi}{2 j+1} P_{j}^{m}(\cos a) \delta_{n j}
$$

in which we invoked Eq.87. This ends the proof.

\subsection{Corollary 8}

For any integers $n, j \in \mathbf{N}$ and any integer $m \in \mathbf{Z}$ :

$$
\begin{aligned}
& \int_{0}^{\pi} \int_{0}^{2 \pi} \overline{Y_{j}^{m}(\theta, \varphi)} P_{n}(\sin a \sin \theta \cos (\varphi-b)+\cos a \cos \theta) \sin \theta d \theta d \varphi \\
= & (-1)^{m} \frac{4 \pi}{2 j+1} Y_{j}^{-m}(a, b) \delta_{n j}=\frac{4 \pi}{2 j+1} \overline{Y_{j}^{m}(a, b)} \delta_{n j}
\end{aligned}
$$

\subsection{Proof of Corollary 8}

Corollary 8 is a simple consequence of Corollary 7. Invoking also Eqs.88-90, we indeed have :

$$
\begin{aligned}
& \int_{0}^{\pi} \int_{0}^{2 \pi} \overline{Y_{j}^{m}(\theta, \varphi)} P_{n}(\sin a \sin \theta \cos (\varphi-b)+\cos a \cos \theta) \sin \theta d \theta d \varphi \\
= & (-1)^{\frac{m-|m|}{2}} \sqrt{\frac{2 j+1}{4 \pi}} \sqrt{\frac{(j-|m|) !}{(j+|m|) !}}
\end{aligned}
$$




$$
\begin{aligned}
& \int_{0}^{\pi} \int_{0}^{2 \pi} P_{j}^{|m|}(\cos \theta) e^{-i m \varphi} P_{n}(\sin a \sin \theta \cos (\varphi-b)+\cos a \cos \theta) \sin \theta d \theta d \varphi \\
= & (-1)^{\frac{m-|m|}{2}} e^{-i m b} P_{j}^{|m|}(\cos a) \sqrt{\frac{4 \pi}{2 j+1}} \sqrt{\frac{(j-|m|) !}{(j+|m|) !}} \delta_{n j}=(-1)^{m} \frac{4 \pi}{2 j+1} Y_{j}^{-m}(a, b) \delta_{n j}
\end{aligned}
$$

\subsection{Additional remarks}

In the series of papers Gouesbet (2006a), Gouesbet (2006b), Gouesbet (2007b), Gouesbet (2007a), one of the integrals required for use in the considered physical issues is Eq.95 of Corollary 8 , with however $b=0$.The second integral required in the same series of papers was given under the following form :

$$
I_{i j}=\int_{0}^{\pi} \int_{0}^{2 \pi} \overline{Y_{i}^{0}}(\sin a \sin \theta \cos \varphi+\cos a \cos \theta) Y_{j}^{0}(\theta) \sin \theta d \theta d \varphi=\left[\sum_{k=0}^{(i-\epsilon) / 2} C_{k i}^{\epsilon}(\cos a)^{2 k+\epsilon}\right] \delta_{i j}
$$

in which :

$$
C_{k i}^{\epsilon}=\frac{(-1)^{(i-\epsilon) / 2}}{2^{i}}(-1)^{k} \frac{(i+2 k+\epsilon) !}{\left(\frac{i-\epsilon}{2}-k\right) !(2 k+\epsilon) !\left(\frac{i+\epsilon}{2}+k\right) !}
$$

where $\epsilon=0,1$ for $i$ even,odd, respectively.

We again use the expansion of Legendre polynomials already invoked at the beginning of the proof of Theorem 4 (Eq.42), and establish that Eq.97 becomes :

$$
I_{i j}(a)=P_{i}(\cos a) \delta_{i j}
$$

Then, by using :

$$
Y_{l}^{0}(\theta)=\sqrt{\frac{2 l+1}{4 \pi}} P_{l}(\cos \theta)
$$

it is easily established that Eq.100 is a special case of Eq.98 (Corollary 8) for $m=0$.

\subsection{Summary}

In the framework of a study examining analogies between electromagnetic and quantum scatterings, the evaluation of two integrals were required. One integral is given by Corollary 8 , for $b=0$, namely :

$$
\begin{aligned}
& \int_{0}^{\pi} \int_{0}^{2 \pi} \overline{Y_{j}^{m}(\theta, \varphi)} P_{n}(\sin a \sin \theta \cos \varphi+\cos a \cos \theta) \sin \theta d \theta d \varphi \\
= & (-1)^{m} \frac{4 \pi}{2 j+1} Y_{j}^{-m}(a, b) \delta_{n j}=\frac{4 \pi}{2 j+1} \overline{Y_{j}^{m}(a, b)} \delta_{n j}
\end{aligned}
$$

The second integral can be obtained as a special case of this result, and can be written as :

$$
\int_{0}^{\pi} \int_{0}^{2 \pi} \overline{Y_{i}^{0}}(\sin a \sin \theta \cos \varphi+\cos a \cos \theta) Y_{j}^{0}(\theta) \sin \theta d \theta d \varphi=P_{i}(\cos a) \delta_{i j}
$$




\section{Conclusion}

Legendre polynomials, associated Legendre polynomials and associated Legendre functions, are widely used in physics, and particularly in light scattering. One of the most famous occurrences of associated Legendre functions is to be found in the Lorenz-Mie theory describing the interaction between an illuminating electromagnetic plane wave and a spherical particle defined by its diameter and its complex refractive index Mie (1908). However, in this theory, only associated Legendre functions $P_{n}^{ \pm 1}$ (or $P_{n}^{1}$ ) do appear. In a generalized Lorenz-Mie theory describing the interaction between an electromagnetic arbitrary shaped beam and (again) a spherical particle defined by its diameter and its complex

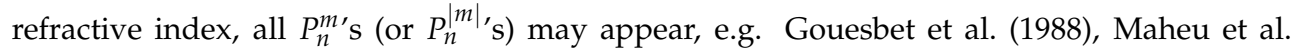
(1988).They actually appear in several places, first of all in the basis functions on which the electromagnetic fields are expanded. Second, expansion coefficients (called beam shape coefficients) can be evaluated by numerical integrations involving the expressions of the electromagnetic fields and associated Legendre functions, e.g. Gouesbet, Lock \& Gréhan (2011). Associated Legendre functions also appear in many expressions generated by the theory, for the evaluation in particular of various cross-sections, under the form of yet other quadratures which, however, may be analytically performed, e.g. appendices in Gouesbet \& Gréhan (2011). Homogeneous spheres defined by a diameter and a complex refractive index are not the only cases of light scattering theories in which associated Legendre functions are involved. They are actually involved whenever the symmetries of the scattering particle require the use of spherical coordinates, such as for multilayered spheres Onofri et al. (1995), assemblies of spheres and aggregates Gouesbet \& Gréhan (1999), or for a spherical particle with an eccentric host sphere Gouesbet \& Gréhan (2000b). May be more surprisingly, associated Legendre functions also play an important role in spheroidal coordinates insofar as, at the present time, beam shape coefficients in spheroidal coordinates are best expressed in terms of beam shape coefficients in spherical coordinates Gouesbet, Xu \& Han (2011). Motivated by the successes of generalized Lorenz-Mie theories and by their numerous applications, an effort has then been devoted to the examination of analogies between electromagnetic arbitrary shaped beams and quantum arbitrary shaped beams, somehow culminating in a generalized optical theorem for non plane wave scattering in quantum mechanics Gouesbet (2009b). During this effort, one of us (G.G) encountered quadratures involving associated Legendre functions which he never encountered before in the framework of generalized Lorenz-Mie theories. This paper provides an analytical evaluation of these quadratures. The reader might be interested in playing with the obtained results, using a symbolic computation software like Maple, as we did to extensively check our derivations.

\section{References}

Abramowitz, M. \& Stegun, I. (1964). Handbook of mathematical functions, Dover Publications, New-York.

G.B.Arfken \& H.J.Weber (2005). Mathematical methods for physicists, sixth edition, Elsevier Academic Press.

Gouesbet, G. (2004). Cross-sections in Lorenz-Mie theory and quantum scattering : formal analogies, Optics Communications 231: 9-15.

Gouesbet, G. (2005). Expansion in free space of arbitrary quantum wavepackets, quantum laser beams, and Gaussian quantum laser beams (on-axis and off-axis) in terms of free spherical waves, Particle and Particle Systems Characterization 22: 38-44. 
Gouesbet, G. (2006a). Asymptotic quantum elastic generalized Lorenz-Mie theory, Optics Communications 266: 704-709.

Gouesbet, G. (2006b). A transparent macroscopic sphere is cross-sectionnally equivalent to a superposition of two quantum-like radial potentials, Optics Communications 266: 710-715.

Gouesbet, G. (2007a). Asymptotically quantum inelastic generalized Lorenz-Mie theory, Optics Communications 278: 215-220.

Gouesbet, G. (2007b). Electromagnetic scattering by an absorbing macroscopic sphere is cross-sectionnally equivalent to a superposition of two effective quantum processes, Journal of Optics A : Pure and Applied Optics 9: 369-375.

Gouesbet, G. (2009a). Generalized Lorenz-Mie theories, the third decade : a perspective, an invited review paper, Journal of Quantitative Spectroscopy and Radiative Transfer, 110, $1223-1238$.

Gouesbet, G. (2009b). On the optical theorem and non plane wave scattering in quantum mechanics, Journal of Mathematical Physics 50: paper 112302, 1-5.

Gouesbet, G. \& Gréhan, G. (1999). Generalized Lorenz-Mie theory for assemblies of spheres and aggregates, Journal of Optics A : Pure and Applied Optics 1: 706-712.

Gouesbet, G. \& Gréhan, G. (2000a). Generalized Lorenz-Mie theories : from past to future, Atomization and Sprays 10 (3-5): 277-333.

Gouesbet, G. \& Gréhan, G. (2000b). Generalized Lorenz-Mie theory for a sphere with an eccentrically located spherical inclusion, Journal of Modern Physics 47,5: 821-837.

Gouesbet, G. \& Gréhan, G. (2011). Generalized Lorenz-Mie theories, Springer.

Gouesbet, G. \& J.A.Lock (2007). Quantum arbitrary shaped beams revisited, Optics Communications 273: 296-305.

Gouesbet, G., Lock, J. \& Gréhan, G. (2011). Generalized Lorenz-Mie theories and description of electromagnetic arbitrary shaped beams : localized approximations and localized beam models, Journal of Quantitative Spectroscopy and Radiative Transfer 112: 1-27.

Gouesbet, G., Maheu, B. \& Grehan, G. (1988). Light scattering from a sphere arbitrarily located in a Gaussian beam, using a Bromwich formulation, Journal of the Optical Society of America A 5,9: 1427-1443.

Gouesbet, G., Xu, F. \& Han, Y. (2011). Expanded description of electromagnetic arbitrary shaped beams in spheroidal coordinates, for use in light scattering theories, a review, Journal of Quantitative Spectroscopy and Radiative Transfer 112: 2249-2267.

J.A.Lock \& Gouesbet, G. (2009). Generalized Lorenz-Mie theory and applications, an invited review paper, Journal of Quantitative Spectroscopy and Radiative Transfer, 110, 800-807 .

Maheu, B., Gouesbet, G. \& Gréhan, G. (1988). A concise presentation of the generalized Lorenz-Mie theory for arbitrary location of the scatterer in an arbitrary incident profile, Journal of Optics, Paris 19,2: 59-67.

Mie, G. (1908). Beiträge zur Optik trüben Medien speziell kolloidaler Metalösungen, Annalen der Physik 25: 377-452.

Onofri, F., Gréhan, G. \& Gouesbet, G. (1995). Electromagnetic scattering from a multilayered sphere located in an arbitrary beam, Applied Optics 34,30: 7113-7124.

Szegö, G. (1939). Orthogonal polynomials, A.M.S. colloquium publications, Vol XXIII, American Mathematical Society, Providence, RI.

T.S.Chihara (1978). An introduction to orthogonal polynomials, Gordon and Breach, New-York. 


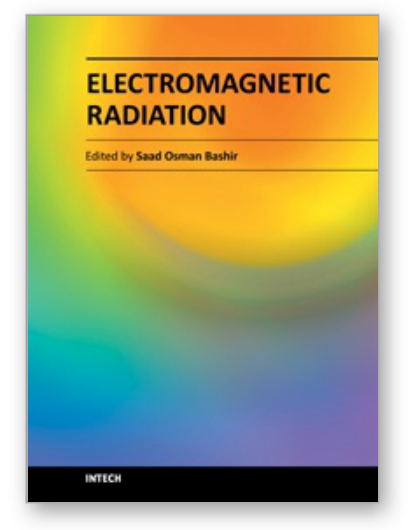

\author{
Electromagnetic Radiation \\ Edited by Prof. S. O. Bashir
}

ISBN 978-953-51-0639-5

Hard cover, 288 pages

Publisher InTech

Published online 05, June, 2012

Published in print edition June, 2012

The application of electromagnetic radiation in modern life is one of the most developing technologies. In this timely book, the authors comprehensively treat two integrated aspects of electromagnetic radiation, theory and application. It covers a wide scope of practical topics, including medical treatment, telecommunication systems, and radiation effects. The book sections have clear presentation, some state of the art examples, which makes this book an indispensable reference book for electromagnetic radiation applications.

\title{
How to reference
}

In order to correctly reference this scholarly work, feel free to copy and paste the following:

A. Draux and G. Gouesbet (2012). Generalized Orthogonality Relation for Spherical Harmonics, Electromagnetic Radiation, Prof. S. O. Bashir (Ed.), ISBN: 978-953-51-0639-5, InTech, Available from: http://www.intechopen.com/books/electromagnetic-radiation/generalized-orthogonality-relation-for-sphericalharmonics

\section{INTECH}

open science | open minds

\section{InTech Europe}

University Campus STeP Ri

Slavka Krautzeka 83/A

51000 Rijeka, Croatia

Phone: +385 (51) 770447

Fax: +385 (51) 686166

www.intechopen.com

\section{InTech China}

Unit 405, Office Block, Hotel Equatorial Shanghai

No.65, Yan An Road (West), Shanghai, 200040, China

中国上海市延安西路65号上海国际贵都大饭店办公楼 405 单元

Phone: +86-21-62489820

Fax: +86-21-62489821 
(C) 2012 The Author(s). Licensee IntechOpen. This is an open access article distributed under the terms of the Creative Commons Attribution 3.0 License, which permits unrestricted use, distribution, and reproduction in any medium, provided the original work is properly cited. 